\title{
SEAWATER DIELECTRIC MEASUREMENTS AT L-BAND WITH LATEST IMPROVEMENTS
}

\author{
Yiwen Zhou ${ }^{1}$, Roger Lang ${ }^{1}$, Emmanuel Dinnat ${ }^{2,3}$ and David Le Vine ${ }^{2}$ \\ ${ }^{1}$ The George Washington University, Washington, DC 20052 USA \\ ${ }^{2}$ Goddard Space Flight Center, Greenbelt, MD 20771 USA \\ ${ }^{3}$ Chapman University, Orange, CA, USA
}

\begin{abstract}
Recently, the dielectric constant of seawater at L-band was determined by employing a resonant cavity technique. A dielectric model function has been developed based on the measurement data and the model function has been used for retrieving the ocean salinity. The results indicate that additional accuracy is still needed to resolve the bias correlated with sea surface temperature. This paper reports the improvements that have been made recently for the development of a more accurate seawater dielectric model function. The additional measurements for the open ocean will be addressed in the paper.
\end{abstract}

Index Terms - Ocean salinity, L-band, Dielectric model function

\section{INTRODUCTION}

An accurate expression for the dielectric constant of sea water as a function of temperature and salinity is important for the remote sensing of sea surface salinity from space. Lang et al. [1] have used a transmission-type cylindrical cavity to determine the complex dielectric constant of seawater accurately at L-band. The real and imaginary parts of the dielectric constant are determined based on the changes in the frequency and Q-value of the cavity before and after the seawater is introduced, respectively. The data has recently been used to construct a model function, $\varepsilon(S, T)$, by fitting the observations to a polynomial [2]:

$$
\varepsilon_{G W}(S, T)=\sum_{i=0}^{3} \sum_{j=0}^{3} p_{i, j} S^{i} T^{j}
$$

where $\varepsilon_{G W}(S, T)$ is the complex dielectric constant; $S$ and $T$ are salinity and temperature, respectively. The coefficients, $p_{i, j}$ are complex numbers that are determined by a leastsquares fit to the data.

The model function has been applied to retrieve the sea surface salinity from the Aquarius satellite data. The comparison between the retrieved salinity and the in-situ data (i.e. Argo floats data) is given in Figure 1. It is seen that the GW model function provides more accurate seawater salinity results at high temperatures --- $25^{\circ}$ to $30^{\circ} \mathrm{C}$ and at low temperatures --- $0.5^{\circ}$ to $7^{\circ} \mathrm{C}$ compared with Klein-Swift model [3] and Meissner-Wentz [4] model.

It can be seen from the figure, however, that more accuracy is still needed for the dielectric model to match the in-situ data with an accuracy better than 0.1 psu. To resolve the bias shown in Fig. 1, additional measurements are being made for seawater at the temperatures typical of the open ocean with an upgraded system. The improvements of the measurement system will also be addressed in this paper.

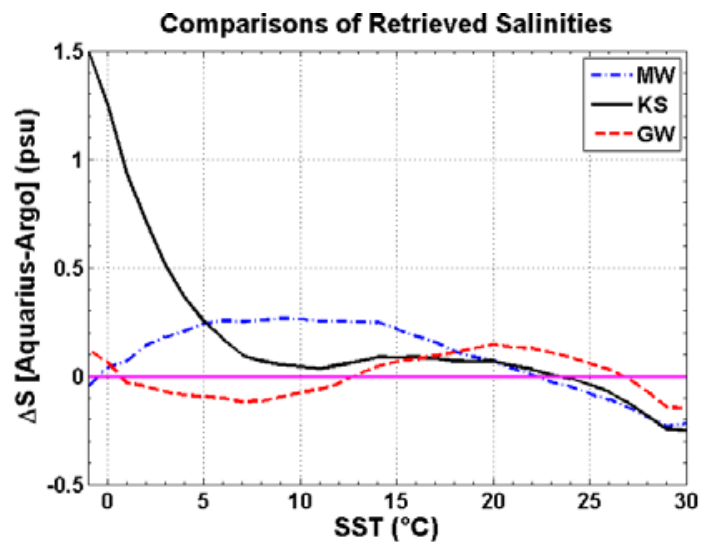

Fig. 1 Difference in-situ salinity (Argo data) and the salinity retrieved using three model functions for the dielectric constant: Klein-Swift (KS), Meissner-Wentz (MW) and GW

\section{NEW SEAWATER MEASUREMENTS AND IMPROVED MEASUREMENT SYSTEM}

\subsection{New Seawater Dielectric Measurements}

The aim of the new seawater dielectric measurements is to resolve the difference between the retrieved salinity and insitu data as shown in Figure 1. It is seen that the greatest bias occurs from $15^{\circ}$ to $25^{\circ} \mathrm{C}$. Figure 2 shows that the frequency of sample occurance as a function of Sea Surface Salinity 
(SSS), offers a possible explanation of the differences. From this figure it is observed that the data used to make Figure 1 comes mainly from salinity values between 34 and 36 psu. GW's measurements, however, were made at 30, 3335 and 38 psu (indicated by red arrows in the figure). Only one of the GW measurments is in the important region between 34 and 36 psu.

To resolve the bias in retrieved salinity for the open ocean region, additional dielectric measurements are being made at the temperature between $15^{\circ} \mathrm{C}$ to $25^{\circ} \mathrm{C}$ by using the seawater sample with 34 psu and 36 psu salinities (indicated by blue arrows in Figure 2). The measurements are made in $5^{\circ} \mathrm{C}$ intervals. At each temperature, at least three measurements are made.

In addition, new measurements are being made at the temperature range between $-1.8^{\circ} \mathrm{C}$ and $5^{\circ} \mathrm{C}$ with a dense grid in temperature. This is because the sensitivity of brightness temperatures to the changes in salinity is very weak [5] at low temperature. The measurement for the cold seawater, as a consequence, requires more accuracy. The seawater sample with 30 psu and 33 psu are used in these measurements since the regions of the ocean associated with cold temperature tend to have lower salinity. These measurement results will be presented at the meeting.

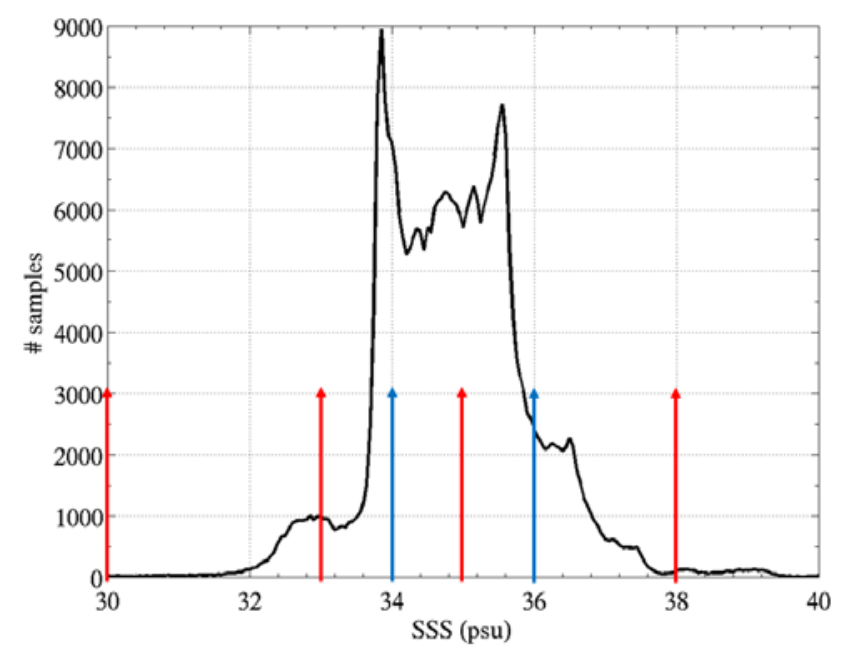

Fig 2: Frequency of occurrence of salinity as observed by Argo. Red Arrows indicate previous GW measurements while Blue Arrows indicate the measurements made in 2018

\subsection{Cavity System Upgrades}

Several upgrades have been made to improve the measurement accuracy. The coaxial RF probes have been replaced with new ones that are TiN coated to prevent corrosion in the coolant. Thermistors with $0.01^{\circ} \mathrm{C}$ accuracy have been purchased and installed. The accuracy of the new thermistors has been validated throughout an extensive temperature range from $-3^{\circ} \mathrm{C}$ to $35^{\circ} \mathrm{C}$. Compared with the old thermistors, they have better accuracy in the important temperature range from $-3^{\circ} \mathrm{C}$ to $0^{\circ} \mathrm{C}$, which will improve the seawater dielectric measurements at low temperature. A new Matlab script has been composed to use the least-square fit to determine the resonant frequency and cavity Q from the raw data collected from the Network Analyzer. This method provides more accurate measurement data.

\subsection{Systematic Error Correction}

To determine the seawater dielectric constant, the system was calibrated using the methanol measurements made at $20^{\circ}$ C. The system has a weak dependence on the temperature. In preparation for the new measurements, an electromagnetic cavity model has been developed that takes into account the changes in the cavity dimensions and the conductivity of cavity walls as temperature changes. The cavity model takes this weak dependence into account and the systematic error due to the calibration is corrected.

Another factor that could affect the measurement accuracy occurs when the tube passes through the center exit-hole on the cavity endplates. This causes a shift in the resonant frequency which results in an error in the real part of the measured dielectric constant [6]. The effect has been examined using a multimode waveguide analysis. Both the seawater and the methanol measurements suffer this frequency shift and they partially cancel one another out. The results due to this effect will be discussed in more details at the meeting.

\section{CONCLUSIONS}

The GW model function has been developed based entirely on data at L-band, but more data is needed to help refine the differences and reduce the error in salinity retrievals. Additional measurements are made with upgraded system to improve the coverage in the range $34 \mathrm{psu}<\mathrm{S}<36 \mathrm{psu}$, which is most common in the open ocean. In the future, the model function will be improved based on the new measurement data and the model function will be applied to retrieve the salinity from the Aquarius and SMAP satellite data.

\section{REFERENCES}

[1] R. Lang, Y. Zhou, C. Utku, and D. Le Vine, "Accurate measurements of the dielectric constant of seawater at L band", Radio Sci., vol.51, pp. 2-24, Jan. 2016

[2] Y. Zhou, R. Lang, E. Dinnat and D. Le Vine, "L-band model function of the dielectric constant of seawater", Trans. Geosci. Remote Sens., vol. 55, no.12, pp. 6964-6974, Dec. 2017

[3] L. Klein and C. Swift, "An improved model for the dielectric constant of sea water at microwave frequencies”, IEEE J. Oceanic Eng., vol. 25, no.1, pp. 104-111, Jan. 1977 
[4] T. Meissner and F. J. Wentz, "The emissivity of the ocean surface between 6 and $90 \mathrm{GHz}$ over a large range of wind speeds and earth incident angles”, IEEE Trans. Geosci. Remote Sens., vol. 50, no. 8, pp. 3004-3026, Aug. 2012

[5] D. M. Le Vine, G. Lagerloef and S.E. Torrusio, “Aquarius and Remote Sensing of Sea Surface Salinity from Space”, Proc. IEEE, vol. 98, no. 5, pp. 688-703, Apr. 2010

[6] Y. Zhou, R. Lang, “Analysis of the composite exit-hole effect on the seawater dielectric measurements”, URSI General Assembly and Scientific Symposium, Montreal, Canada, Aug. 2017 